\title{
The Network Spinal Wave as a Central Pattern Generator
}

\author{
Simon A. Senzon, MA, DC, Donald M. Epstein, DC, and Daniel Lemberger, DC ${ }^{3}$
}

\begin{abstract}
Objectives: This article explains the research on a unique spinal wave visibly observed in association with network spinal analysis care. Since 1997, the network wave has been studied using surface electromyography (sEMG), characterized mathematically, and determined to be a unique and repeatable phenomenon.

Methods: The authors provide a narrative review of the research and a context for the network wave's development.

Results: The sEMG research demonstrates that the movement of the musculature of the spine during the wave phenomenon is electromagnetic and mechanical. The changes running along the spine were characterized mathematically at three distinct levels of care. Additionally, the wave has the mathematical properties of a central pattern generator $(\mathrm{CPG})$.

Conclusions: The network wave may be the first CPG discovered in the spine unrelated to locomotion. The mathematical characterization of the signal also demonstrates coherence at a distance between the sacral to cervical spine. According to mathematical engineers, based on studies conducted a decade apart, the wave itself is a robust phenomenon and the detection methods for this coherence may represent a new measure for central nervous system health. This phenomenon has implications for recovery from spinal cord injury and for reorganizational healing development.
\end{abstract}

\section{Introduction}

$\mathbf{T}$ HIS PAPER EXPLORES AN EMERGENT spinal wave phenomenon termed, and herein referred to as, the network wave. The network wave involves a visible undulation and specific rocking movement of spinal segments, which is elicited through gentle contacts in a defined sequence to the spine at specific areas. The areas are associated with the location of meningeal attachments of the spinal cord to the vertebral column.

The sequencing and application are aspects of network spinal analysis (NSA) care (network care). Network care is practiced by chiropractors using network spinal analysis protocols developed by Donald Epstein. ${ }^{1,2}$ The Network wave was first clinically demonstrated in 1987, and has been studied since 1997 at several major universities and institutions. ${ }^{3}$

The novelty and repeatability of the network wave led to empirical research in the fall of 1998 and the spring of 1999 by Edmond Jonckheere and his team at the University of Southern California in the Department of Electrical Engineering and Mathematics. ${ }^{4}$ Jonckheere and his colleagues studied the network wave through the use of surface electromyography (sEMG) to measure the electrical changes in the muscle system. ${ }^{4}$ Since 1998 , sEMG has been used as a window into the central nervous system. ${ }^{4-16}$ Epstein developed the NSA protocols based on clinical practice and in response to the research into the network wave.

Initially, the sEMG signal was analyzed to determine the mathematical characteristics of the network wave. ${ }^{4}$ An example of how the wave is understood by examining the sEMG data is described in Figure 1, which demonstrates some of the data. Figure 1 shows the result of the wavelet decomposition (using the waviest filter) of two signals. ${ }^{15}$ The one on the left is the test signal, and the one on the right is the signal from the network wave. The results of the filtering show a difference in the underlying, seemingly noisy, signal at level 7 and level 8 of the decomposition.

The researchers then analyzed the sEMG signal to determine whether the network wave had different attributes based upon the NSA level of care. ${ }^{5}$ NSA care includes three distinct levels of care. Of this finding Jonckheere wrote in 2009, "the objectively established fact that the signals become less

\footnotetext{
${ }^{1}$ The Institute Chiropractic, Asheville, NC.

${ }^{2}$ Association for Reorganizational Healing Practice, Washington, DC.

${ }^{3}$ Epstein Technologies, Longmont, CO.
}

(C) Simon A. Senzon et al., 2016; Published by Mary Ann Liebert, Inc. This Open Access article is distributed under the terms of the Creative Commons Attribution Noncommercial License (http://creativecommons.org/licenses/by-nc/4.0/) which permits any noncommercial use, distribution, and reproduction in any medium, provided the original author(s) and the source are credited. 

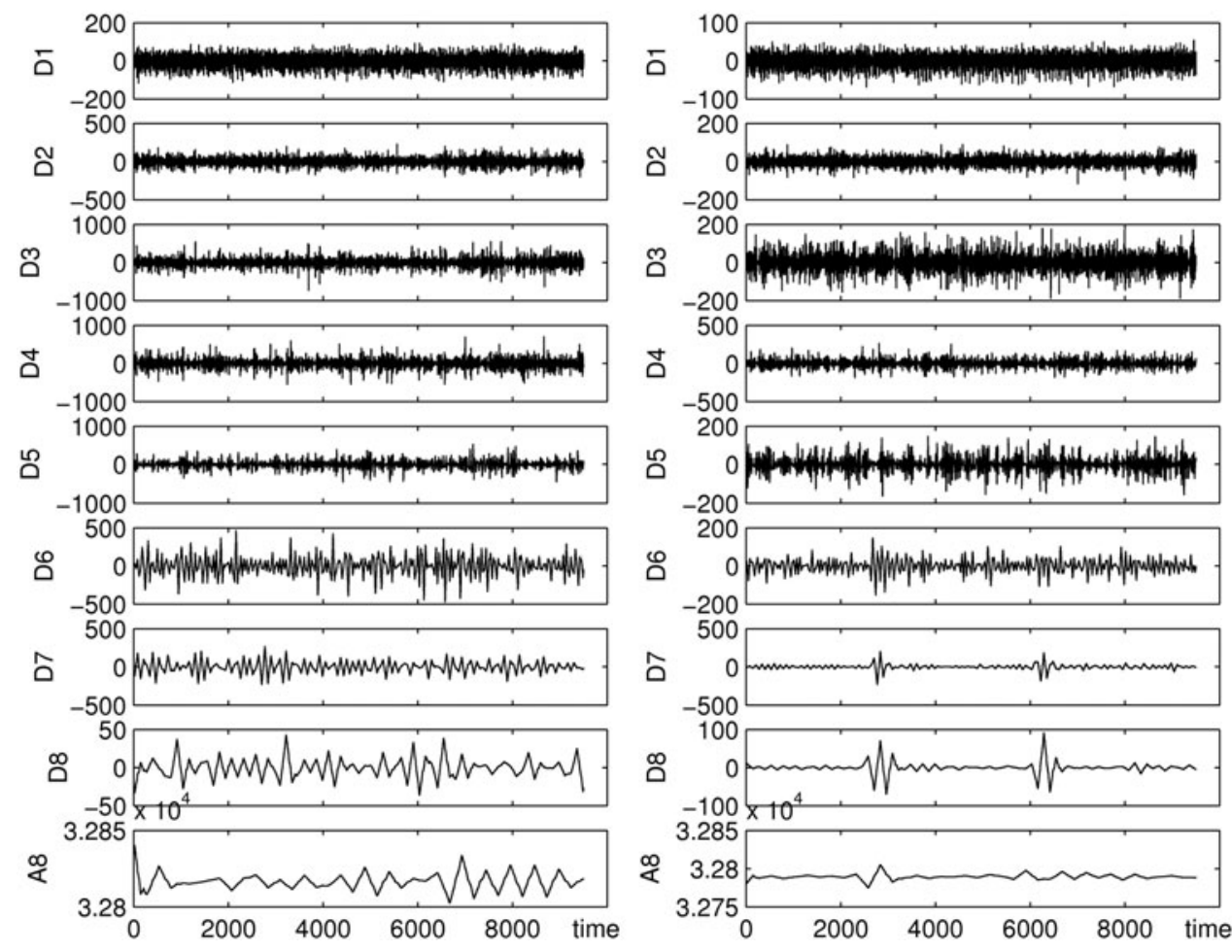

FIG. 1. Demonstrating wavelet packets (D7 and D8) and coherent bursting phenomena as a standing wave running up and down the spine. The left side is the test signal and the right side is the spinal wave signal. Figure reprinted with permission from Jonckheere et al. On a standing wave central pattern generator and the coherence problem. Biomed Signal Process Control; Elsevier; 2010.

random and more predictable can be interpreted to reveal a better 'organization' of the neural circuitry at advanced levels of care." 17 Thus, the researchers found that the sEMG signal gets more organized with the higher levels of network care.

Other research was developed to distinguish differences between an initial electrical burst detected in the musculature and the background noise of the sEMG signal. ${ }^{7}$ To explore this distinction new mathematical approaches were developed.

By 2004, the sEMG signal of the network wave was best described by using mathematical modeling for a central pattern generator (CPG). ${ }^{12}$ CPGs are well described in the literature in relation to locomotion, such as walking, swimming, and flying. However, this was the first time a CPG was observed in the spine apart from locomotion or respiration. ${ }^{12,13,15}$ Furthermore, while other known CPGs originate in the lumbar and thoracic spine, the neurologic circuitry for the network wave originates from the sacral and cervical spine. ${ }^{15}$

The NSA protocol emphasizes the cervical and sacral areas based on a clinical analysis system, which characterizes five phases of adverse mechanical spinal cord tension (AMCT). ${ }^{18}$ The tension is associated with patterns of defensive physiology and intermittent areas of relaxation and ease. ${ }^{2}$ The defense physiology associated with AMCT is assessed by examining the stabilizing spinal subsystems (muscular, bony, and neural). ${ }^{19,20}$ Structural rigidity associated with defensive postures may lead to a static nonadaptable state, which limits the experience of novelty, and constructive change for the individual. Such a state is an inefficient use of energy. In response to the spinal contacts from the practitioner, over time, the spinal system moves from defense physiology, which is characterized by areas of muscle facilitation of the spine, decreased respiration, and other indicators of adverse mechanical cord tension (AMCT), into a state promoting the emergent network wave properties. ${ }^{20}$ The decreased spinal cord tension is a precursor to the development of the network wave.
The spinal wave associated with network care has been described as "an electrophysiological phenomenon running along the spine,", 15 created by "a sensory-motor loop instability," 12 which stabilizes into unique oscillatory patterns. ${ }^{16}$ The dynamic movement patterns then reorganize into more complex behavioral wave movements, ${ }^{17}$ "settling in a Central Pattern Generator.", 12 The development of the wave itself suggests a process of neurologic entrainment. The specific light-touch contacts to the spine at three unique levels of network care act as repeated stimuli leading to the entrainment of oscillators or vertebral motion segments moving in rhythmic and synchronous patterns. Development of the network wave may take several weeks to several months for an individual patient.

In describing the spinal wave associated with network care, the researchers, comprising mathematicians and electrical engineers, wrote,

The overall spinal wave procedure consists of the following steps: After sensitization of the sacral area, a light pressure contact at S3-S4 engages the sacral oscillator. From the sacral area, an electrophysiological wave phenomenon propagates upward, but initially dissipates before reaching the cervical area. Nevertheless, after some entrainment, eventually the upward wave reaches the cervical area and triggers the neck area to go in oscillation. When extended across the whole spine, the headward traveling wave reflects off the sphenoid, which happens to be the most cephalad attachment of the dura, and then travels caudally. Visually, the upward/ downward traveling waves can be seen to collide, and survive the collision in some soliton-like propagation. Eventually the upward and downward waves settle in a standing wave pattern, during which the neck movement is perfectly coordinated with the pelvic movement. ${ }^{15}$

With this rich evidence base ${ }^{5-11,13,16,17,21}$ along with other research and clinical developments, ${ }^{10,22-30}$ Epstein 
used the research data to systematically refine how the network wave develops in the human nervous system. ${ }^{20}$

A recent presentation by Martin-del Campo and Jonckheere explored two distinct experiments that were spaced 10 years apart and included different participants, changes to software and instrument configurations, different positioning of sacral electrodes, different sEMG amplifiers, and different analog-todigital conversion methods. ${ }^{31}$ Even with all of these complex factors, they concluded that "the results upon which the Central Pattern Generator hypothesis rests are reproducible" and that "the spinal wave is a coherent movement elicited by a Central Pattern Generator, opening the road for the potential of this coherence analysis to become part of the neurological suite." ${ }^{31}$ This latest research points to reproducibility of the network wave and the sEMG data it generates and also the fact that coherence, which indicates a healthy functioning nervous system, emerges along with the CPG.

Epstein hypothesized that the bound energy released from the defensive patterns associated with adverse spinal cord tension becomes available as energy to create a higher-order spinal and neural integrity. ${ }^{20}$ This hypothesis may help to explain why the wave increases in complexity of oscillation over time and with advancing levels of care. ${ }^{17}$ It may also explain the emergence of coherence at a distance between the cervical and sacral spine. ${ }^{31}$ The coherence is an aspect of the CPG, which emerges causally from the NSA protocols. These results point not only to the first CPG apart from locomotion in the spine but also to a healthier and more organized nervous system.

\section{Methods}

This article is an overview of empirical research on the network wave, which was originally called the somatopsychic wave. The findings of the research into the network wave are presented in an accessible manner to health care practitioners and researchers, simplified and translated from the mathematical explanations of the engineers. For complete technical descriptions and schematics, see several publications in the fields of engineering, mathematics, and information processing theory. ${ }^{9,10,32}$ The evolution of the network wave research is explored from a historical perspective by describing several pivotal discoveries. This article concludes with a discussion of AMCT, CPG, soliton waves, boundary conditions, spinal coherence, and the spinal gateway region because these concepts further help to explain the network wave phenomenon.

\section{The Preliminary Research}

Findings indicated that the somatopsychic waveform (an early name for the network wave) possessed a "nonlinear 'attractor.', 4 This initial research was undertaken to determine the mathematical characteristics of the wave, more specifically whether it demonstrated nonlinear and chaotic attributes. The research demonstrated that the network wave could be explained with greater predictability using nonlinear mathematical models as opposed to linear models. Figure 2 shows the results of comparing nonlinear and linear statistical models obtained from the time series analysis of the EMG signals. The result shows higher correlations for the nonlinear models when compared with the linear models (Fig. 2). ${ }^{4}$

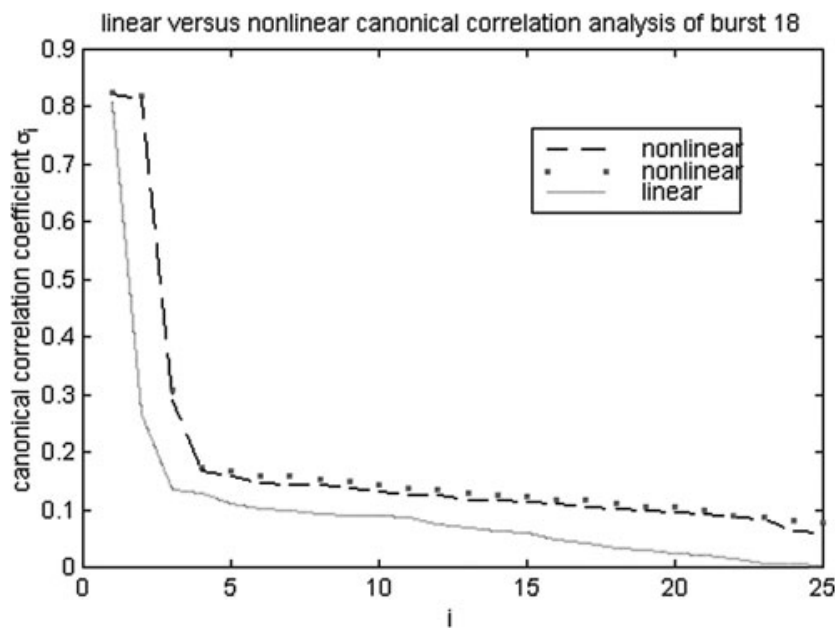

FIG. 2. Linear and nonlinear research - linear and nonlinear canonical correlation coefficients for surface electromyography of the waveform associated with network spinal analysis. The key difference between the plots is that the second coefficient is much larger in the case of the nonlinear canonical correlation. This is an indicator that some nonlinearity is present. Figure reprinted with permission from Bohacek et al. Chaotic modeling in network spinal analysis; J Vert Subl Res; 1998.

While the more traditionally known linear models suggest that a small change in initial conditions of a system results in a proportionate modification of the system, nonlinearity is associated with what is considered a dynamic shift. This shift is one in which a small change in a system can result in an immediate or delayed disproportionate reorientation of the system or its outcomes. The classic example is how the flap of a butterfly's wings could set in motion drastic changes in weather patterns. ${ }^{33}$ Living systems are also considered dynamic and may thus be described using nonlinear models. Epstein developed network care by treating the healing process as one that was nonlinear, whereby a small input such as the contact from the practitioner would cause a global and dynamic response in the spinal system and the individual's health-related quality of life. ${ }^{20}$

Several interesting observations were made during this early research: There was commonality between amplitude and frequency of the wave signal across participants; the wave was deemed involuntary yet could be stopped at any time; and upon stopping, there was no modification of the wave, just dissipation. As noted earlier, bursts of electromyographic activity were found with the sEMG sensors. Figure 3 demonstrates that nonlinear prediction models were more accurate to explain the sEMG bursts than linear models. ${ }^{4,34}$ These bursts were determined to be the kind of attractors found in dynamic systems. ${ }^{31}$ An attractor may be described as a point or a form toward which the system evolves. ${ }^{35}$

\section{Objective Measures of Levels of Care}

In May 1999, research began to focus on level-specific changes. $^{36}$ The researchers sought to determine whether different wave characteristics exist based specifically on the level of network care application (Table 1).,

One of the defining features of each level of care in the NSA protocols includes a specific and rhythmic pattern of 

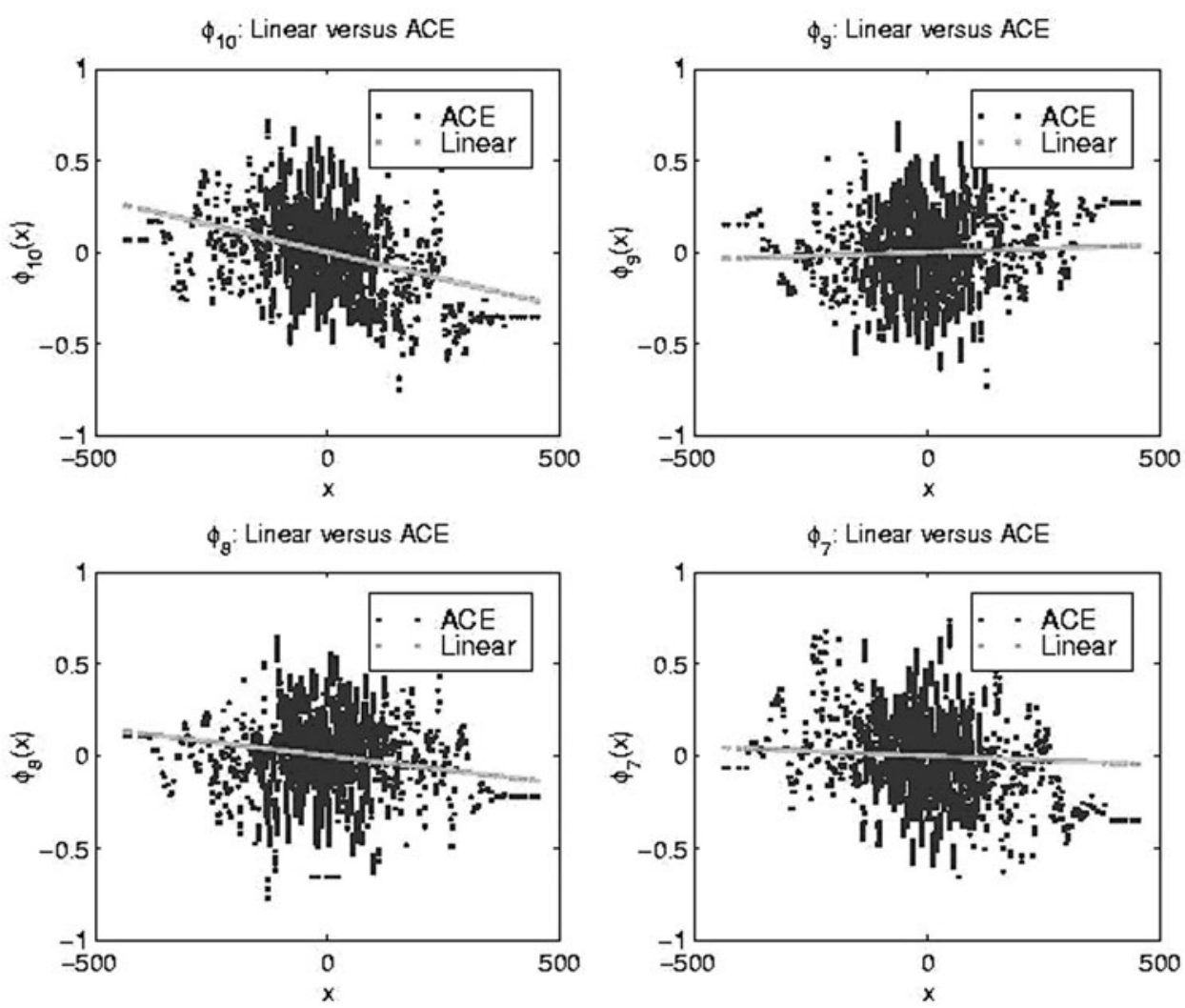

FIG. 3. Bursts of wave activity measured with surface electromyography sensors using a nonlinear canonical correlation analysis coupled to a specific implementation referred to as alternating conditional expectation (ACE) to determine nonlinearity. Figure reprinted with permission from Bohacek et al. Chaotic modeling in network spinal analysis; J Vert Subl Res; 1998. movement of the vertebra. When the vertebra move in such a rhythmic manner, the movement may be described as an oscillation and the vertebral motion segments as oscillators. The motion of the oscillators increases in complexity with each level of care: that is, synchronous

Table 1. Network Spinal Analysis Levels of Care AND LeVEL-SPECIFIC Clinical OUtcomes

Level 1 Awareness and entrainment of respiratory motion with spinal motion (respiratory wave), reduction of parameters of adverse spinal cord tension, release of tension from spinal stability subsystems, enhancement of spinal and neural integrity, reorganization of spinal structures, development of body's ability to self-regulate tension, increase in basic somatic awareness

Level 2 Stretching of areas of spinal tension, resolution of dominant spinal defense patterns, development and refinement of the network wave (entrainment of two vertebral oscillators) temporally and spatially through the spinal gateway, advancement of client's somatic and self-awareness

Advanced Development of network wave with care standing wave between front and back of body, absence of defensive physiology, development of the third (thoracic/heart area) oscillator, synchronized and refined entrainment of three oscillators, shift in awareness and consciousness movement develops in multiple spinal oscillators simultaneously over time. Each level of care is characterized by a new level of complexity of the wave along with the clinical findings, which indicate coupling and coherence between spinal integrity subsystems (muscles, bones, and nerves). ${ }^{19}$

Research conclusions indicate a characteristic burst activity of the sEMG signals set amidst a background activity. These bursts were studied as dynamic phenomenon. ${ }^{5}$ The consistency of the dynamic properties of the bursts was a significant and meaningful finding at this point. ${ }^{5}$ The fact that there was a larger-magnitude level of organization of the burst in advanced care (level 3) as compared with levels 1 or 2 directly leads to the conclusion that the data "provide(s) an early mathematical confirmation that there is something objective in the concept of 'Levels of Care.,",5 The sEMG signal at higher levels of care has a distinctly higher "level of organization" compared with the lower levels. ${ }^{5}$ Frequency and amplitude of the bursts increase with level of care. Figure 4 depicts a segment of sEMG data when the spine transitions from a level 2 response to a level 3 response. $^{36}$

The "bursty activity" leveled off or quieted during level 2. ${ }^{5}$ This led Epstein to revise the level 2 protocols. ${ }^{20}$ The newer NSA protocols were developed to further entrain the network wave response in the spine. The new objective was to create transient instability of the stabilizing energy and information associated with defensive strategies. This was done in part by stretching the specific spinal segments to release stored tension in the connective tissues associated with the spinal joint. The released tension is used by the body in more visible movement after the stretch is complete. The increased energy and information could then become 
FIG. 4. Transition between level 2 and level 3. Figure reprinted with permission from Jonckheere et al. Dynamical Modeling in Network Spinal Analysis (NSA) Care; 2003.

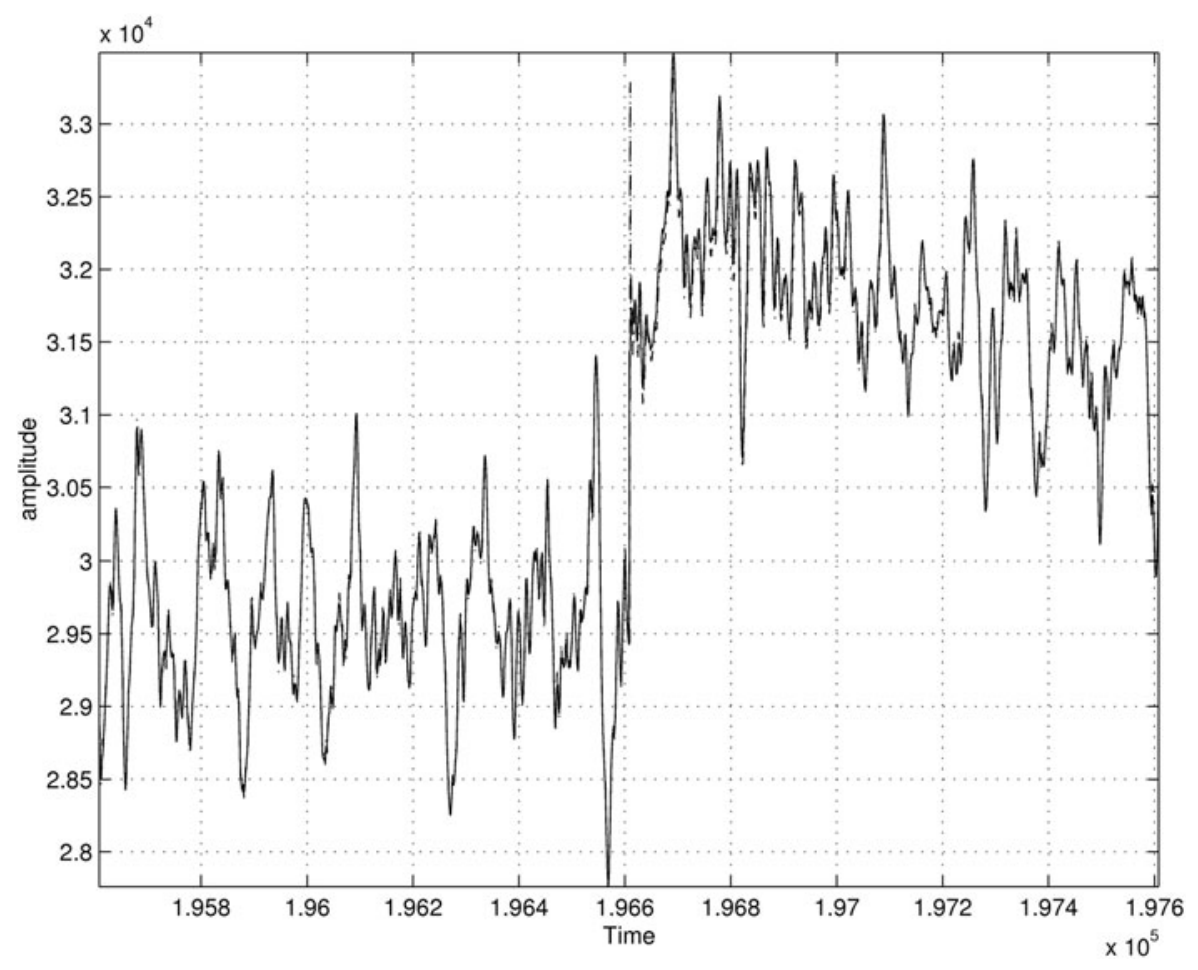

available to produce precise segmental oscillation linked to the spinal standing wave. ${ }^{20}$

\section{Development of New Mathematical Models to Describe New Complex Behaviors}

Jonckheere and colleagues then focused on other aspects of the signal. They wanted to explore the fact that the signal had self-similarity, which is characteristic of dynamic sys- tems. To do this they examined the possibility that the electrical burst characteristic of the network wave and the background noise of the sEMG signal were part of a "global dynamical phenomenon." 6 The new research focus involved a characterization of the standing wave in the spine by including new mathematical approaches.

The new mathematical models were initiated to correlate the burst activity with the background. The question explored was which part of the signal was most important in terms of
FIG. 5. Full neck surface electromyography signal. Figure reprinted with permission from Lohsoonthorn et al. Nonlinear switching dynamics in surface electromyography of the spine; Physics and Control Proceedings; 2003.

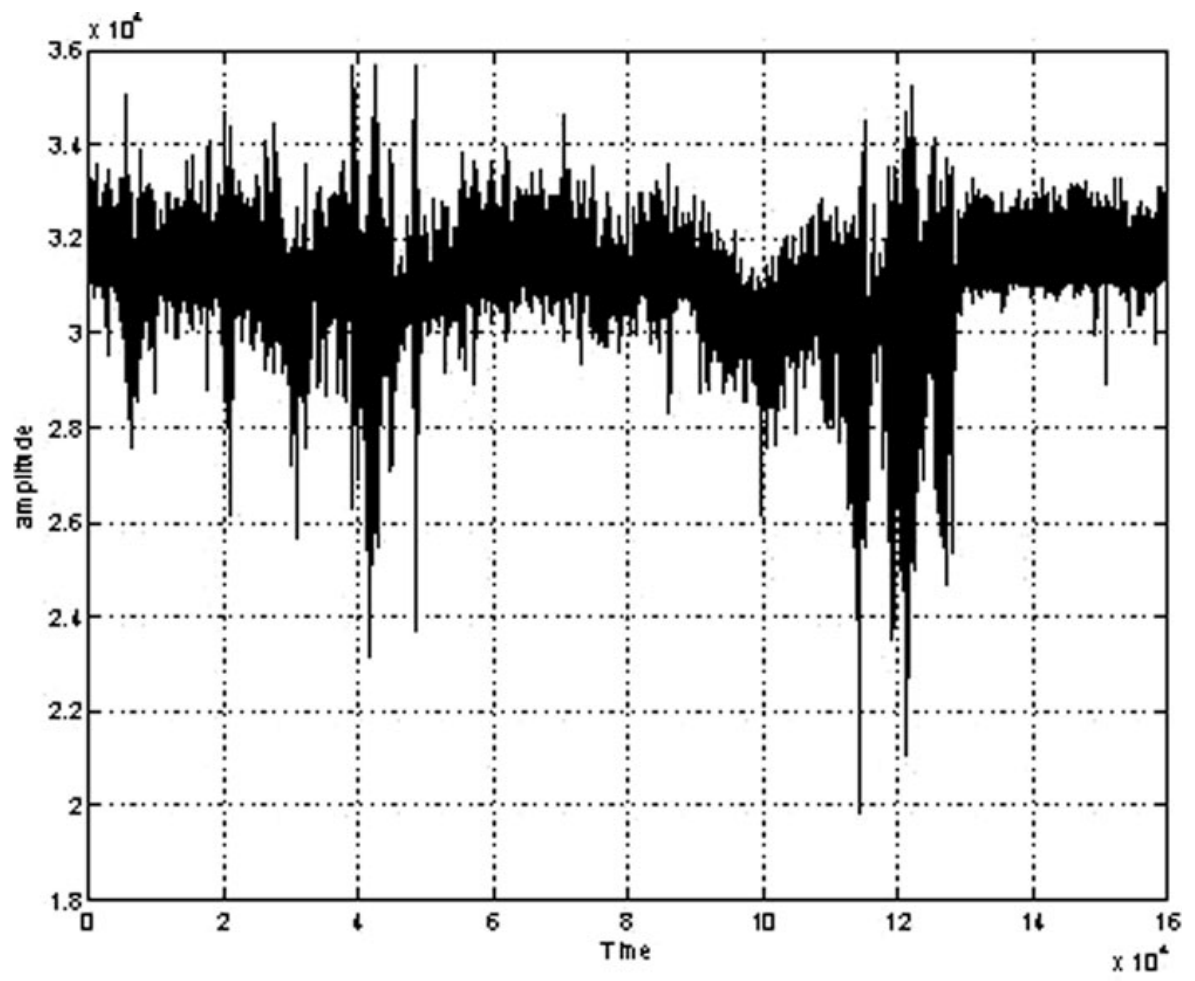




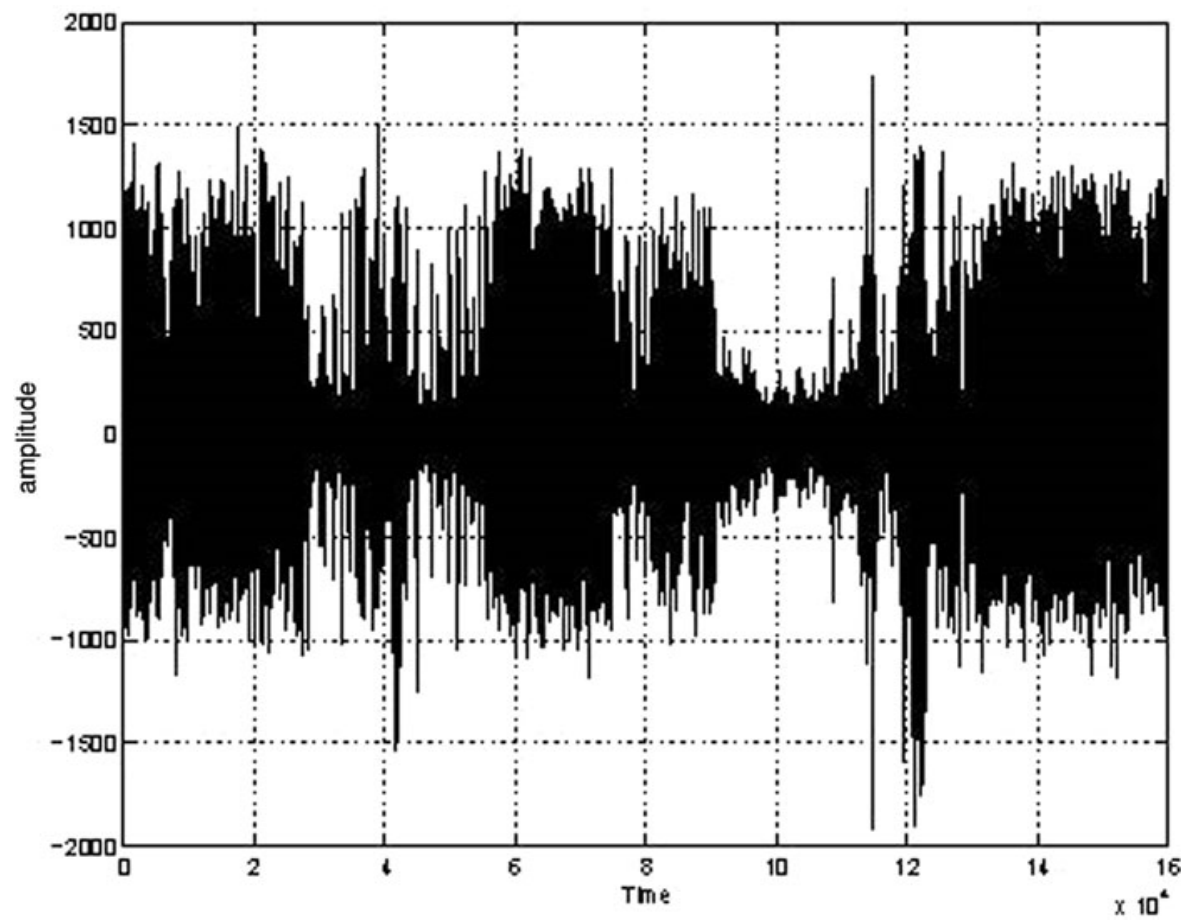

FIG. 6. Incremental neck surface electromyography signal. Figure reprinted with permission from Lohsoonthorn et al. Nonlinear switching dynamics in surface electromyography of the spine; Physics and Control Proceedings; 2003.

carrying information relevant to spinal and neural integrity and health. ${ }^{7}$ To the eye, it seemed that the initial bursts indicated a build-up of wave activity. Figure 5 shows sEMG data (amplitude versus time) collected from the sensor at the neck. This "raw" signal is then analyzed to determine the underlying dynamics that result in the NSA wave. Figure 6 shows an incremental signal (i.e., the signal obtained by finding the difference between the consecutive points). This is a standard preprocessing done in statistical time series analysis to remove certain unwanted features (Figs. 5 and 6). ${ }^{9}$

A significant difference was determined between the burst and the background. ${ }^{7}$ Both were deemed "a new class of

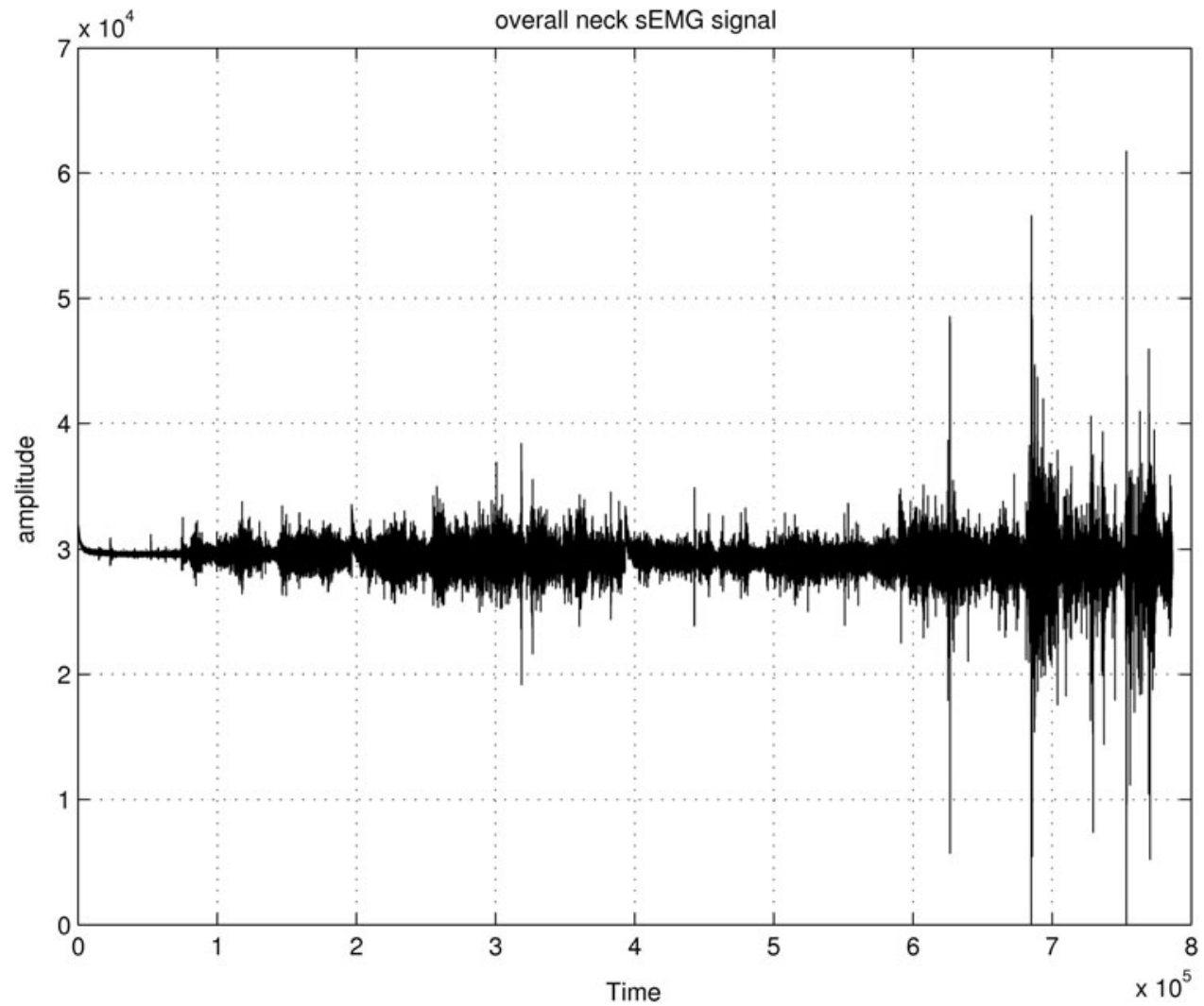

FIG. 7. Raw signal of beginning background becoming burst. Figure reprinted with permission from Jonckheere et al. Dynamic modeling of spinal electromyographic activity during various conditions; American Control Conference; 2003. 
complex nonlinear dynamic behavior." 9 The behavior was considered novel because the nonlinear switching between the burst and the background involved a specific frequency. The frequency was higher than the peak of the background. Figure 7 represents an sEMG segment that shows a transition between two qualitatively different subsegments. The "background" is distinguished from the "burst" by a sudden increase in the amplitude as seen at the right end (Fig. 7). ${ }^{8}$

Other models of switching neurodynamics reveal frequency shifts but not oscillation at higher frequency. ${ }^{37,38}$ Jonckheere and colleagues suggest similarities with burst activity of the thalamus, pointing to further research. ${ }^{7}$ This is especially relevant because the network wave demonstrated some involvement with brain activity in a preliminary functional magnetic resonance imaging study. ${ }^{8}$

The new mathematical approaches were also designed to study the space-time interval of the network wave. The goal was to assess the relationship between the point of practi- tioner's contact on the spine and the spinal response, which was often at the opposite end of the spine.

\section{Network Wave as CPG}

Several factors led to the successful modeling of the network wave as a CPG. The first factor was based on research that a quadriplegic patient was able to experience the network wave below (past) the area of injury with some recovery of sensory and motor functions after NSA care. ${ }^{12}$ The wave did travel through the injury but did not settle into a stationary wave, such as in a healthy person. Thus, the network wave does not necessarily require higher cortical input. Because the wave may continue to oscillate without further input, it displays a central characteristic of a CPG. Further input may modulate the wave but research has shown that it is not necessary to sustain it. ${ }^{12,13,15}$

Figures 8 and 9 is the result of the special Euclidean extension analysis that compares the features of the data from
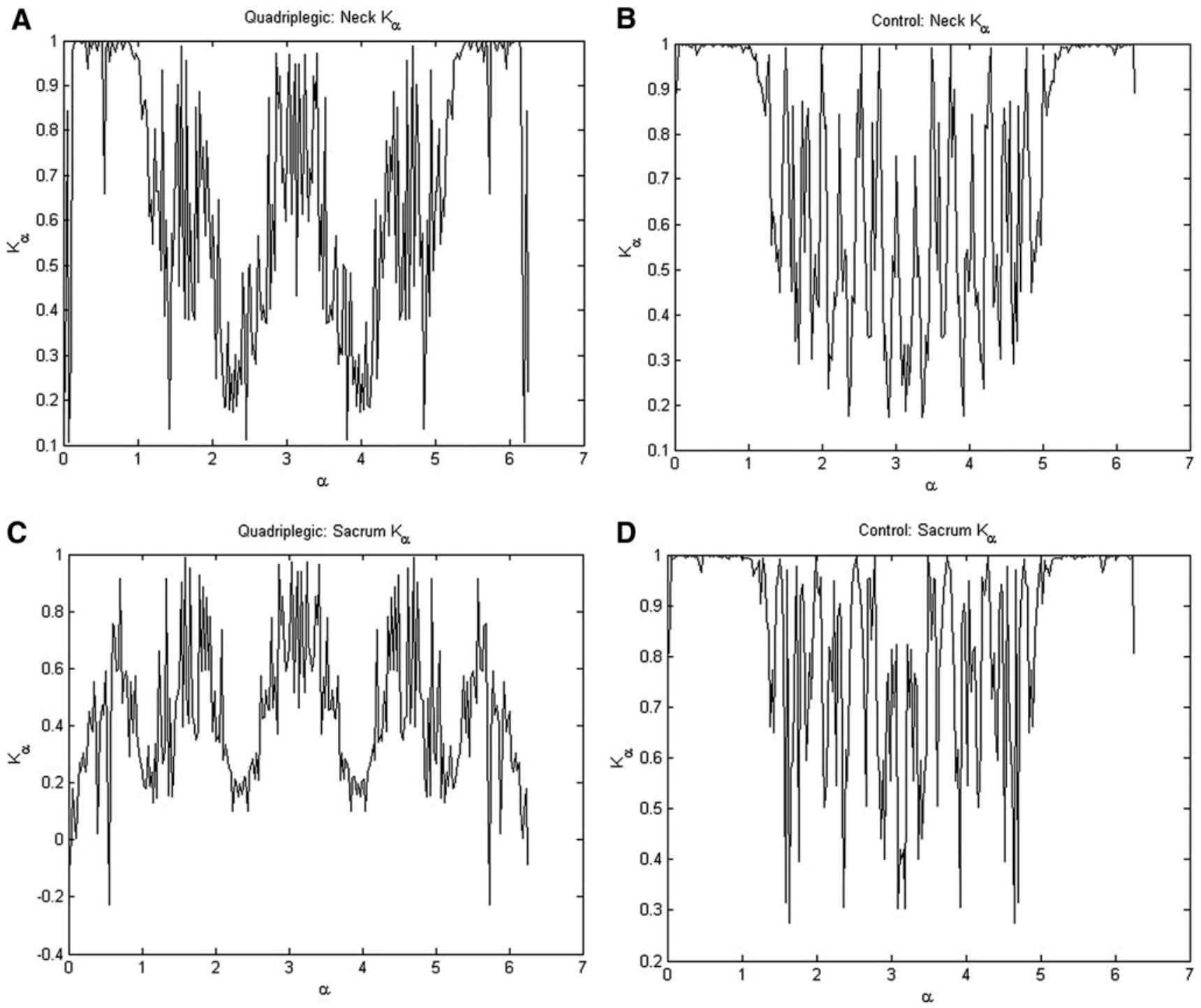

FIG. 8. Coherence between neck and sacrum: quadriplegic versus healthy: case 1. (A) Quadriplegic neck. (B) Healthy neck. (C) Quadriplegic sacrum. (D) Healthy sacrum. Figure reprinted with permission from Musuvathy. Coherently interacting dynamics in the neuromuscular system; 2011. 
A
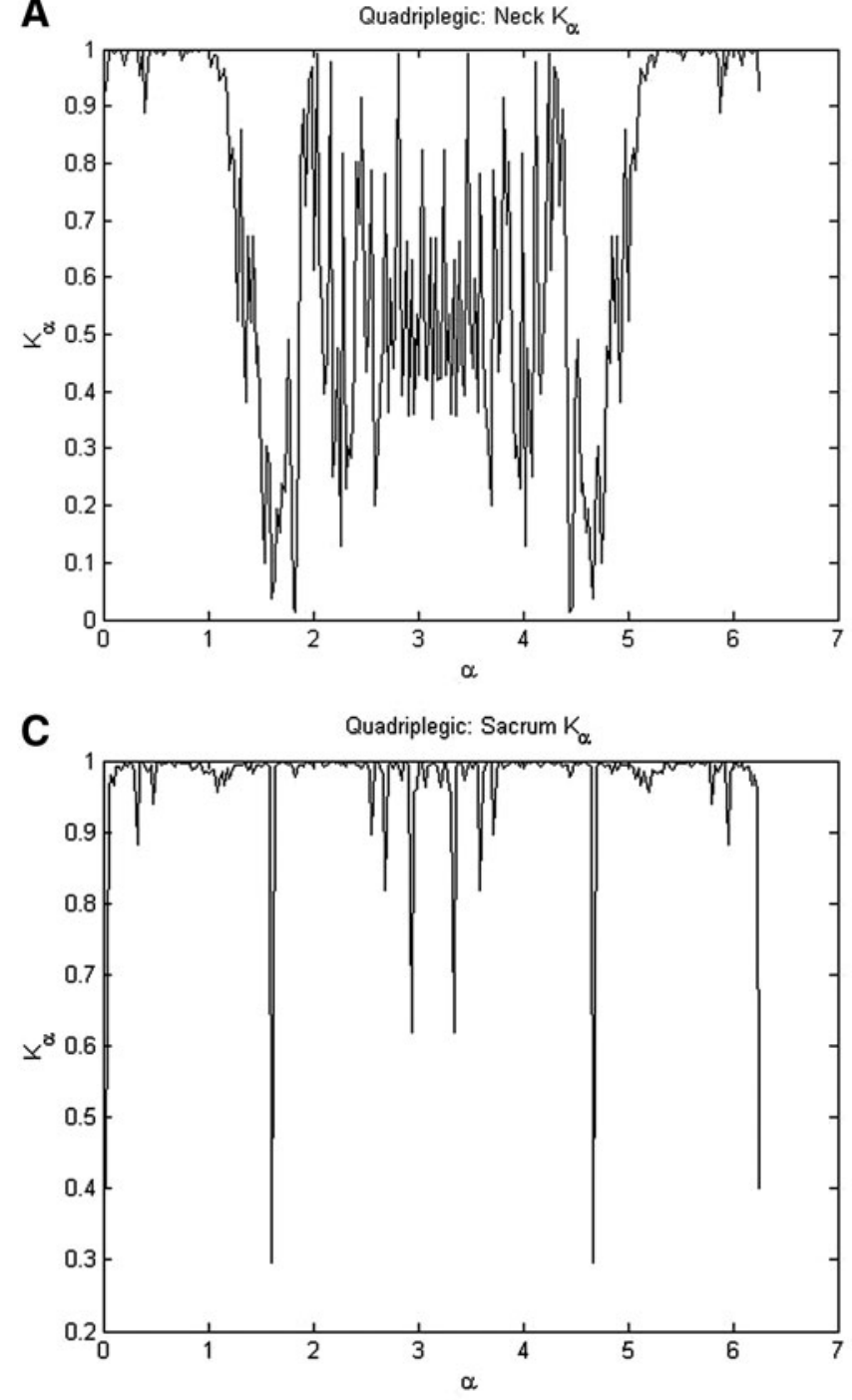

B

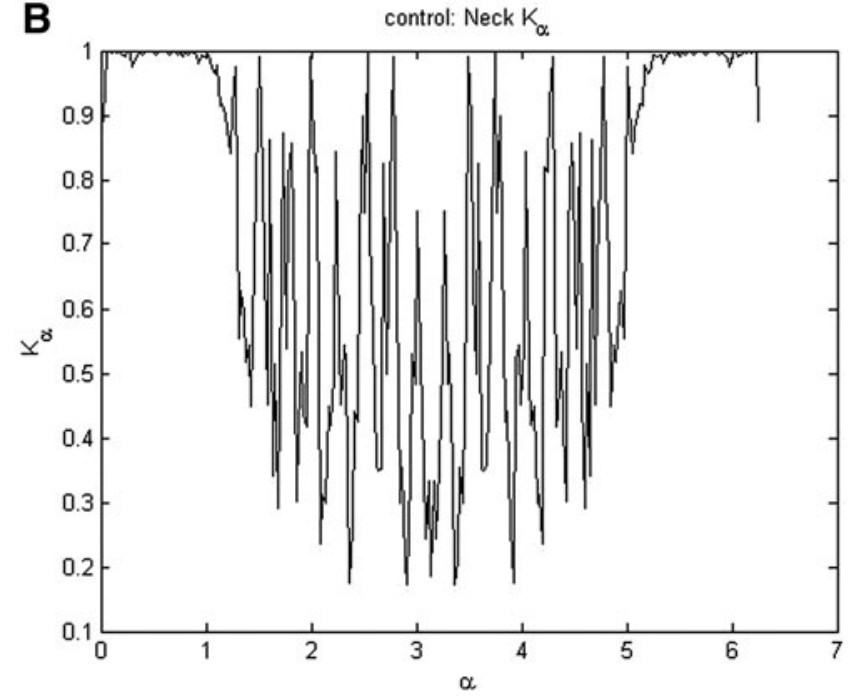

D

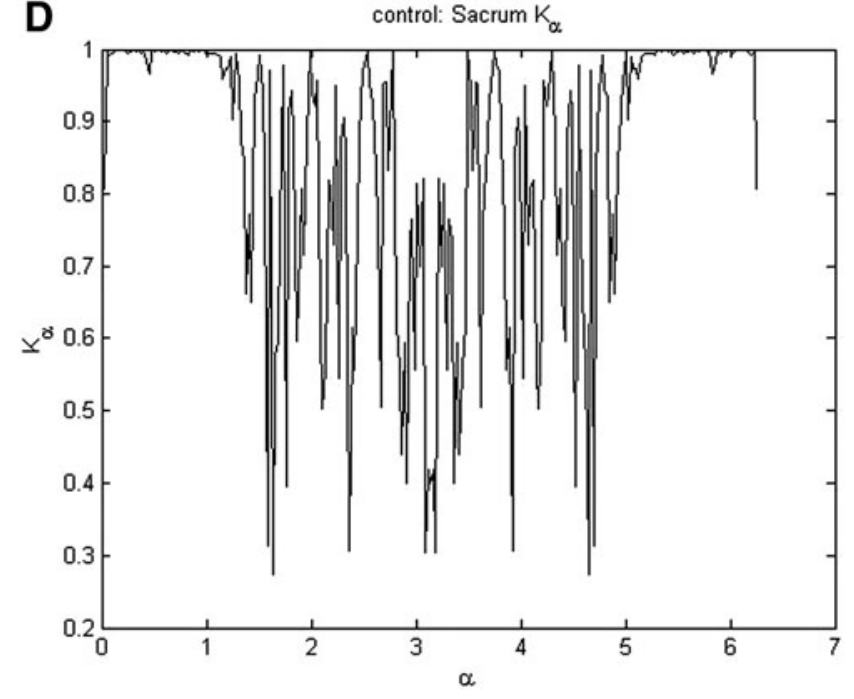

FIG. 9. Coherence between neck and sacrum: quadriplegic versus healthy: case 2. (A) Quadriplegic neck. (B) Healthy neck. (C) Quadriplegic sacrum. (D) Healthy sacrum. Figure reprinted with permission from Musuvathy. Coherently interacting dynamics in the neuromuscular system; 2011.

the neck and sacrum during a level 2 wave. The plots show the presence of power (roughly the average squared amplitude) at various frequencies. Values close to 1 indicate presence of power at that corresponding frequency. Values close to 0 indicate an absence. When compared, healthy persons demonstrate similar patterns of power versus frequencies, thus indicating coherence. Participants with spinal cord injuries show an absence of good coherence (Figs. 8 and 9). ${ }^{46}$

The bursting signals, which increase with the levels of care, suggest a synchronization of neuronal firing. With this model, as noted above, Jonckheere et al. were able to more precisely predict levels of care based on position and bursting or background activity. They suggest the network wave is learned through Hebbian learning, learning that occurs when neural circuits emerge from activity that is correlated. 5,12,38,39

Although the electromagnetic bursting patterns based on the sEMG data are similar to the burst patterns of other $\mathrm{CPGs}^{15}{ }^{15}$ significant differences stand out. The neurologic circuits of locomotion (walking) and rhythmic arm movement (swimming) occur in the segmental oscillators of the thoracolumbar junction and the cervical-thoracic junction, respectively. ${ }^{39}$ The circuitry of the wave, however, seems to originate in the upper cervical spine and the sacrum, or areas of spinal cord attachments. Figures 10 and 11 show the possible feedback diagram of the circuitry at the neck and sacrum that could explain the oscillations. The feedback from the muscles (plants in control theory) via the sensors is processed by the controllers. These feedbacks, with the "right" gains, can result in oscillations (Figs. 10 and 11). ${ }^{15}$ This circuit was suggested by Jonckheere and colleagues based on Breig's paradigm of adverse mechanical cord tension. ${ }^{18}$

The conjecture that AMCT, as proposed by Breig, which increases with postural tension, as the filum terminale acts like a stretching "rubber band" 12 also led to a view of the network wave as a CPG. The gentle stretching of the cord at the filum terminale and coccyx allows for lengthening of the cord on flexion and extension, which could lead to the adverse tension that may create a sensory-motor instability. ${ }^{12}$ 
FIG. 10. Sensory motor loop at cervical level. The hard lines represent established pathways and the dotted lines are conjectured paths closing the loop. Figure reprinted with permission from Jonckheere et al. On a standing wave central pattern generator and the coherence problem. Biomed Signal Process Control; Elsevier; 2010.

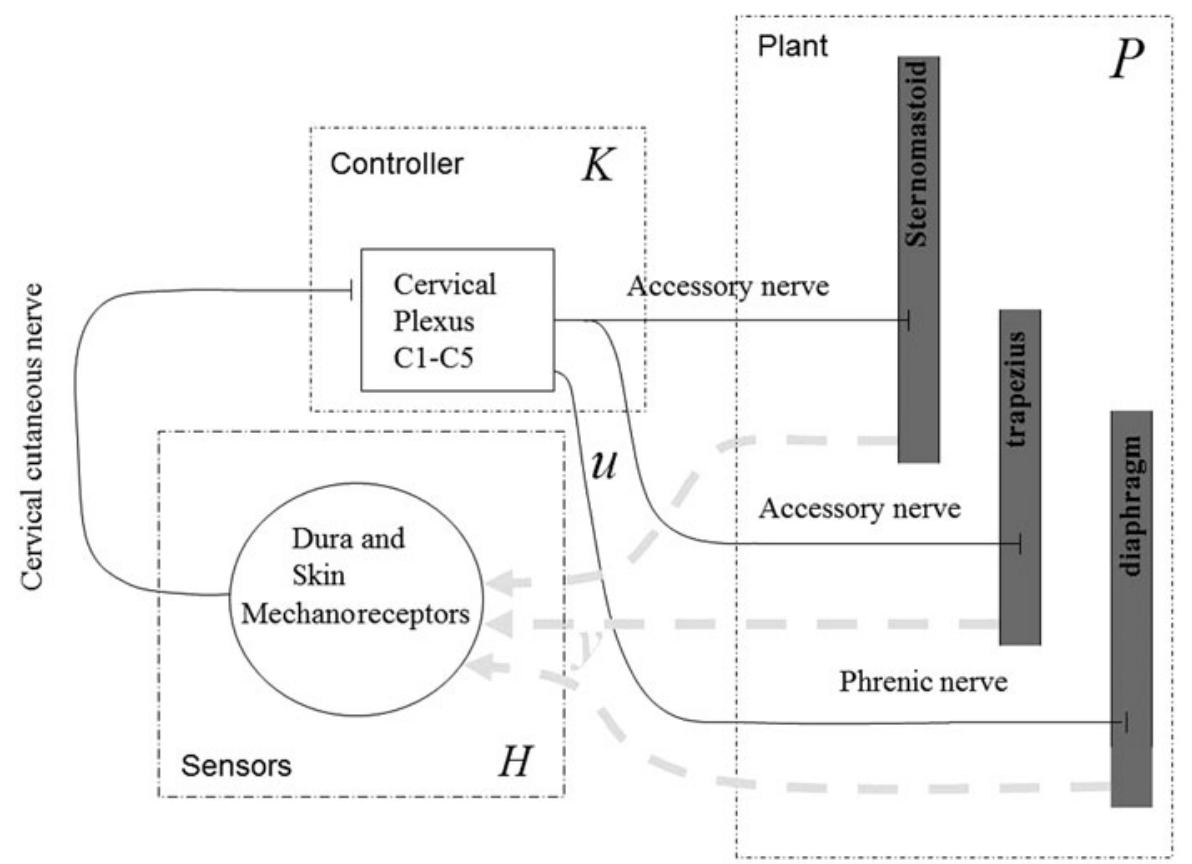

Jonckheere and colleagues proposed that the dural attachments at the cervical spine and sacrum create additional pathways to the classic motor reflex loop of mechanical movement of the spinal muscles to the central nervous system. The classic motor reflex loop includes the stretching of paraspinal muscles, which are recorded by neuromuscular spindles. This information is transmitted by the afferent nerves to the motor neurons in the spine and then transmitted back to the muscles by the efferent nerves. An extra path is created by the dural attachments from the paraspinal muscles to the spinal neurons directly. This path closes the loop. They write, "The existence of this feedback loop has been demonstrated by the spinal oscillators, which can be elicited by a technique referred to as Network Spinal Analysis care.",12

The adverse mechanical cord tension may create a sensory-motor instability, which upon entrainment settles into an oscillation. The oscillation propagates first as a traveling wave from the cervical and sacral areas and settles into a standing wave, which is a CPG. There may be additional explanations of this phenomenon, including a sensory-motor loop formed by alpha and gamma motor neurons, or the formation of other reflex loops. ${ }^{40}$ The research demonstrates the network wave is the first known
FIG. 11. Sensory motor loop at sacral level. The solid lines represent established pathways and the dotted lines are conjectured paths closing the loop. Figure reprinted with permission from Jonckheere et al. On a standing wave central pattern generator and the coherence problem. Biomed Signal Process Control; Elsevier; 2010.

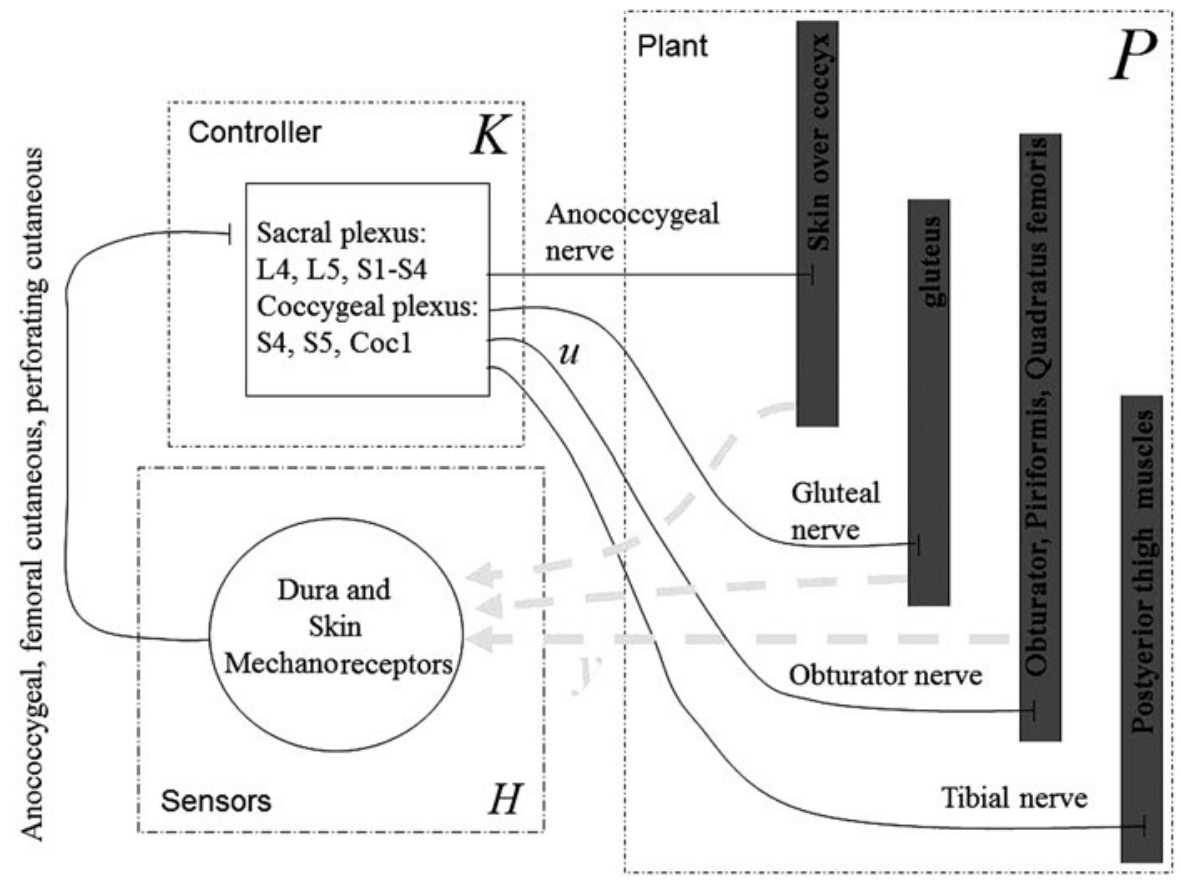


segmental oscillatory CPG in humans unrelated to locomotion or arm movement. ${ }^{15}$

\section{Discussion}

This discussion includes several elements that led to these discoveries of the network wave as a CPG, including the Breig paradigm, a more complete understanding of CPGs, the importance of boundary conditions, the properties of soliton waves, coherence in the spinal and neural system, and Epstein's development of the spinal gateway region as the point of contact for the spinal entrainment in the NSA protocols.

\section{Breig's paradigm}

Jonckheere et al. mainly relied on Breig's neurosurgery paradigm, which has been fundamental since 1985 in Epstein's teaching, ${ }^{41}$ for the models of neurologic circuitry to account for these unique phenomena. The dural attachments from the foramen magnum, through the arch of $\mathrm{C} 1$, with varying attachments from $\mathrm{C} 2$ to $\mathrm{C} 7$, and again at the coccyx with the filum terminale, create an extra feedback loop on the path from the paraspinal muscles to the spinal nerves. They conclude that this neurological circuit is demonstrated by the network care spinal entrainment, also termed network adjustment, and the responding oscillation. The conjecture of an additional feedback loop, apart from the standard muscle mechanoreceptor to proprioceptor model, offers one possible mechanism by which the spinal wave propagates and sustains itself. ${ }^{7,8}$

\section{CPG}

The central nervous system generates and coordinates patterns of forces that are dynamic and rhythmic. ${ }^{39,42}$ CPGs organize rhythmic movement patterns of synchronized or "entrained" oscillators. The best-known CPGs are respiration, flight, running, walking, and swimming. These behavior patterns can be sustained without direct sensory input and without higher cerebral involvement. Thus, a CPG is a rhythmic movement of neuronal centers that may continue without requiring further movement or sensory feedback to remain self-sustaining. ${ }^{39}$

The CPG model unifies two strands of neurologic theory from the 20th century. ${ }^{43}$ The first strand is Sherrington's theory that neural origins of movement are coordinated by the central nervous system as a combination of sensory feedback and reflexes. ${ }^{40}$ The second strand is Brown's model of inhibitory control circuits as the source of rhythmic patterns. ${ }^{44}$ Both theories converge in the $\mathrm{CPG}$, wherein rhythmogenesis may be explained along "general principles of pattern generation." 45 Some oscillatory neurons inherently couple in synchrony. The temporal and spatial synchrony between synapses of CPGs generally exists within inhibitory circuits. Rhythmogenesis emerges because "rhythmic elements" are found within the circuitry and embedded in the spinal cord. ${ }^{39}$

\section{Boundary conditions and soliton waves}

The sacrum and cervical connections to the dura create a boundary condition whereby the spine can act as a "propagation medium with sensory motor loops." 12 The wave reflects on the ends of the spine, creating a traveling wave that settles into a stationary wave. Figure 12 is a sample of sEMG data. The shades (between 2 and 4 and around 12) show a low-amplitude quiet region and a high-amplitude burst region. $^{46}$ (In the more recently collected data, the changes are much smoother and the distinction is not obvious. ${ }^{31}$ ) The cervical area has many more connections to the dura and is thus highly complex. It may be the origin of the wave. ${ }^{12}$

Viewing the ends of the spine and their oscillatory nature as a boundary condition offers a "missing piece of the puzzle" to CPG research and theory. ${ }^{14}$ The fact that the network waves move from the neck down to the sacrum and from the sacrum up to the neck and collide, but do not disperse, suggests the waves propagate as solitons. Soliton waves, like tidal waves, sustain energy and information. The spinal soliton waves then bifurcate into specific regionally located oscillations.

This is congruent with Epstein's original conjectures that the spinal contact from the practitioner acts as a boundary condition for the wave to emerge and that the spine may act as a dissipative structure. ${ }^{47}$ It also points to other models of nerve pulse propagation ${ }^{48,49}$ and biologically closed electronic circuits. ${ }^{50}$

\section{Coherence in the network wave}

The sustained oscillation at the neck and sacrum demonstrates coherent dynamics in the spine. Coherent behavior is viewed as indicating a healthy spine ${ }^{16}$ and greater strength

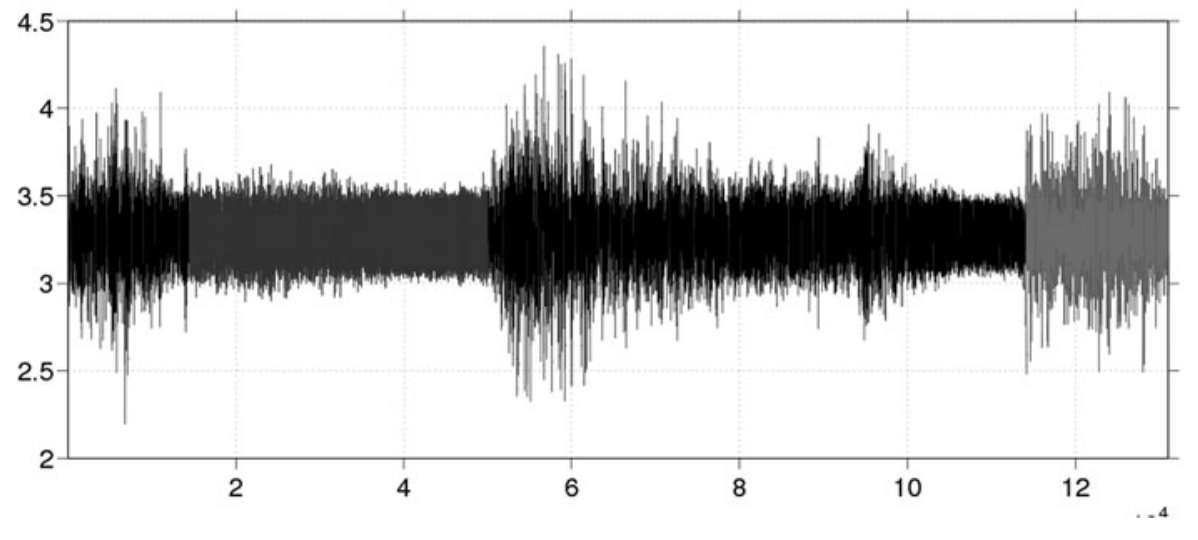

FIG. 12. sEMG data collected from the cervical region during standing wave. Figure reprinted with permission from Musuvathy. Coherently interacting dynamics in the neuromuscular system; 2011. 
and complexity of the synaptic connections. ${ }^{17}$ This coherence also demonstrates an evolution over time and an increase in the organization of the neural circuitry. ${ }^{17}$

The coherence observed in the spinal wave may be a result of greater synchronization of the two oscillators. The soliton, which is the result of the interaction of the spinal waves, may help in this synchronization. Coherence was demonstrated only with healthy spines. Coherence was not demonstrated in persons with spinal cord injury. This points to the possibility of self-organizing behavior in a dynamic system. $^{46}$

Interestingly, when a person attempts to reproduce the motion of the wave, without a specific network care application the motion visually looks similar to or even identical to the network wave; however, it lacks the unique mathematical coherence characteristic of this phenomenon. Put simply, the higher self-organization as seen with the NSA care happens only with the network applications and does not occur with faked similar movements. Of further importance, the linked precise vertebral oscillation for each level of care is a significant predictor of the coherence and self-organization characteristic of the level of care. ${ }^{51}$

\section{Spinal gateway region}

After 2000, the electromyographic readings were different from the readings prior to 2000, which is likely because of Epstein's introduction of the spinal gateway region. The initial studies were characterized by "bursting" of the spinal musculature during the wave process. ${ }^{7}$ The more recent data indicate less bursting and greater self-organization of the background, settling into a central pattern generator in the spine. ${ }^{46}$ A hypothesis is that it was the introduction of the spinal gateway region in the clinical protocol that resulted in a greater efficiency of the application and thereby a more specific response of the nervous system.

Epstein postulated that the spinal gateway regions are generally located in areas of spinal cord attachments; that they are regions of probability within the intersegmental connective tissues; and that these regions modulate between stress, tension, and available energy. ${ }^{52}$ Epstein proposed this new structure by combining insights from the dynamic mathematical modeling of the network spinal wave, ${ }^{4}$ with research by Jones and Bae into the acoustic nature of acupoints, ${ }^{53}$ along with several other clinical and theoretical approaches. ${ }^{52}$ The spinal gateway is related to physiology, anatomy, and subtle energy anatomy characteristic of energy medicine.

\section{Conclusions}

The circuitry of a CPG embedded in the spine generating the network wave points to new areas of CPG research, spinal cord injury research, as well as novel research into the spine as a mediator of pattern generation, which may dissipate adverse mechanical spinal cord tension (AMCT). ${ }^{18}$ The dissipation of AMCT relates to a wide range of possible research foci from spinal cord injury on the therapeutic end of the spectrum and emergent wellness lifestyles on the other end. The emergence of wellness lifestyles has been linked to a reorganization of the spinal behaviors and structures coupled to novel perceptions. ${ }^{24}$
The spinal wave associated with network care has been well documented. It appears that each level of care is organized by unique central organizing principles mediated by the network wave through frequency entrained oscillation. The level of care reflects increasing levels of coherence and energy efficiency of the spinal subsystems organized through a CPG, demonstrated by distinct changes/differences in the frequency and amplitude of the bursts with respect to each level of network care (level 1, level 2, advanced). Overall, the wave implies an endogenous reorganizational system, which exists in the range between the stress and relaxation systems. ${ }^{54,55}$ It also relates to the dynamic push and pull between stability and instability associated with far-from-equilibrium living systems. ${ }^{56}$

The network wave may produce a temporary reorganizational instability to the defensive physiology in the spinal system. Through this process, the wave may help the individual to achieve a higher level of adaptable stability in their nervous system organization.

The network wave occurs at a higher self-organizational threshold, in the absence of significant adverse mechanical cord tension, and with enhanced self-regulation of the spinal subsystems. With the onset of central pattern generation, modulated through the network wave, reorganizational behavior may emerge in the individual's spine and life as a whole.

\section{Author Disclosure Statement}

No competing financial interests exist.

\section{References}

1. Epstein D. Network spinal analysis: a system of health care delivery within the subluxation-based chiropractic model. J Vertebral Subluxation Res 1996;1:1-9.

2. Epstein D, Senzon S, Lemberger D. Reorganizational healing: a paradigm for the advancement of wellness, behavior change, holistic practice, and healing. J Altern Complement Med 2009;15:475-487.

3. Senzon S, Lemberger D. A history of network spinal analysis. Association for the History of Chiropractic Annual Conference. Cinncinatti, OH, June 4-7, 2009.

4. Bohacek S, Jonckheere E. Chaotic modeling in network spinal analysis: preliminary report. Nonlinear canonical correlation with alternating conditional expectation (ACE). J Vert Subl Res 1998;2:188-195.

5. Jonckheere E, Bohacek S. Chaotic modeling in network spinal analysis: mathematical classification of levels 1, 2, 3 patients. Los Angeles: Department of Electrical Engineering and Department of Mathematics, University of Southern California; 2000.

6. Jonckheere E, Lohsoonthorn P. Dynamical modeling in network spinal analysis: self-similar data analysis and spatial correlation. Los Angeles: Department of Electrical Engineering Systems, University of Southern California; 2000.

7. Jonckheere E, Lohsoonthorn P. Dymamical modeling in network spinal analysis: bursting versus background mode discrimination. Los Angeles: Department of Electrical Engineering Systems, University of Southern California; 2002.

8. Jonckheere E, Lohsoonthorn P, Boone W. Dynamic modeling of spinal electromyographic activity during various 
conditions. Session WA- 13-3, Biomedical Applications. Presented at American Control Conference; Denver, CO; June 4-6, 2003.

9. Lohsoonthorn P, Jonckheere E. Nonlinear switching dynamics in surface electromyography of the spine. Physics and Control Proceedings. International Conference. Vol 1. 2003:276-281.

10. Jonckheere E, Lohsoonthorn P. Spatio-temporal analysis of an electrophysiological wave phenomenon. Presented at International Symposium on the Mathematical Theory of Network and Systems; Leuven, Belgium; July 5-9, 2004.

11. Jonckheere E, Lohsoonthorn P, Mahajan V. ChiroSensor: an array of noninvasive sEMG electrodes. Medicine Meets Virtual Reality. Vol 111. Long Beach, CA, Amsterdam: IOS Press, Technology and Informatics; 2005:234-236.

12. Jonckheere E, Lohsoonthorn P, Mahajan V. Sensory motor instability and central pattern generator in spinal oscillations. Los Angeles: Department of Electrical Engineering and Department of Mathematics, University of Southern California; 2005.

13. Hiebert A, Jonckheere E, Lohsoonthorn P. Visualization of a stationary CPG-revealing spinal wave [poster presentation]. Medicine Meets Virtual Reality. Vol 119. Long Beach, CA: Amsterdam: IOS Press; 2006:198-200.

14. Jonckheere E, Musuvathy S, Stefanovic M. A biologically inspired synchronization of lumped parameter oscillators through a distributed parameter channel. Control Distributed Parameter Syst 2007:87.

15. Jonckheere E, Lohsoonthorn P, Musuvathy S, et al. On a standing wave central pattern generator and the coherence problem. Biomed Signal Process Control 2010;5:336-347.

16. Musuvathy S, Jonckheere E. Evidence of spatio-temporal transition to chaos in the spine. Presented at 4th International Symposium on Communications, Control and Signal Processing. 2010: 1-5.

17. Jonckheere E. Network spinal analysis. J Altern Complement Med. 2009;15:469-470.

18. Breig A. Adverse Mechanical Tension in the Central Nervous System: An Analysis of Cause and Effect: Relief by Functional Neurosurgery. New York: Almqvist \& Wiksell International; 1978.

19. Panjabi M. The stabilizing system of the spine. Part I. Function, dysfunction, adaptation, and enhancement. J Spin Disord Techniques 1992;5:383-389.

20. Epstein D. Theoretical Basis and Clinical Application of Network Spinal Anaylsis (NSA) and Evidence based Document, rev. xi. Longmont, CO: Innate Intelligence; 1995/2005.

21. Boone W. The evidenced based evolving model of network spinal analysis [abstract]. Presented at Association for Network Care: Scientific Research Conference; Como, Italy; November 17-18, 2001.

22. Kidoo K. The role of network spinal analysis in augmenting psychotherapy [abstract]. Presented at Association for Network Care: Scientific Research Conference; Como, Italy; November 17-18, 2001.

23. Senzon S. Successful in vitro fertilization in a poor responder while under network spinal analysis care: a case report. J Vertebral Subluxation Res 2003(Sept 14):1-6.

24. Schuster T, Dobson M, Jauregui M, Blanks R. Wellness lifestyles II: modeling the dynamics of wellness, health lifestyle practices, and network spinal analysis. J Altern Complement Med 2004;10:357-367.

25. Pauli Y. Improvement in attention in patients undergoing network spinal analysis: a case series using objective measures of attention. J Vertebral Subluxation Res 2007; August23:1-9.

26. Miller E, Redmond P. Changes in digital skin temperature, surface electromyography, and electrodermal activity in subjects receiving network spinal analysis care. J Vertebral Subluxation Res 1998;2:1-9.

27. Epstein D. The transition of network spinal analysis care: hallmarks of a client-centered wellness education multicomponent system of health care delivery. J Vertebral Subluxation Res 2004;5:1-7.

28. Blanks R, Schuster T, Dobson M, Jaurequi M. Assessment of network spinal analysis in retrospective and prospective research design formats using a survey of self-reported health and wellness [abstract]. Presented at Association for Network Care: Scientific Research Conference; Como, Italy; November 17-18, 2001.

29. Blanks R, Schuster T, Dobson M. A retrospective assessment of network care using a survey of self-rated health, wellness and quality of life. J Vertebral Subluxation Res 1997;1:11-27.

30. Behrendt M. Reduction of psoriasis in a patient under network spinal analysis care: a case report. J Vertebral Subluxation Res 1998;4:1-5.

31. Martin-del Campo R, Jonckheere E. Stationary regime for standing wave central pattern generator. Presented at 3rd IEEE Global Conference on Signal \& Information Processing; Orlando, FL; December 14-16, 2015.

32. Jonckheere E, Lohsoonthorn P, Musuvarthy S, et al. On a standing wave central pattern generator and the coherence problem. Biomed Signal Process Control 2010;5:336-347.

33. Lorenz E. The Essence of Chaos. London: Routledge; 1995.

34. Jonckheere E, Bohacek S, Lohsoonthorn P. Dynamic modeling of spinal EMG activity. Presented at NSF Southwest Regional Workshop on New Directions in Dynamical Systems; November 16-19, 2000; University of Southern California, Los Angeles.

35. Combs A. Radiance of Being: Understanding the Grand Integral Vision; Living the Integral Life. 2nd ed. St. Paul, MN: Paragon House; 2003.

36. Jonckheere E, Lohsoonthorn P, Boone W. Dynamical Modeling in Network Spinal Analysis (NSA) Care: Level of Care Specificity of sEMG Signals at Cervical, Thoracic, Lumbar, and Sacral Spinal Segments. Los Angeles: Department of Electrical Engineering and Department of Mathematics, University of Southern California; 2003.

37. Koch C. Biophysics of Computation: Information Processing in Single Neurons. New York: Oxford University Press; 2004.

38. Kopell N. We got rhythm: dynamical systems of the nervous system. Notices of the AMS. 2000;47:6-16.

39. Falgairolle M, de Seze M, Juvin L, et al. Coordinated network functioning in the spinal cord: an evolutionary perspective. J Physiol Paris 2006;100:304-316.

40. Sherrington $\mathrm{C}$. The integrative action of the nervous system. Cambridge, UK: Cambridge University Press Archive; 1966.

41. Epstein D. The stress connection: gauging the role of the nervous systm. Digest Chiropract Econ 1987;Dec/Jan:58-60.

42. Eliasmith C, Anderson C. Rethinking central pattern generators: a general approach. Neurocomputing 2000;32:735740.

43. Friesen W, Cang J. Sensory and central mechanisms control intersegmental coordination. Curr Opin Neurobiol 2001;11: 678-683. 
44. Brown T. On the nature of the fundamental activity of the nervous centres; together with an analysis of the conditioning of rhythmic activity in progression, and a theory of the evolution of function in the nervous system. J Physiol 1914;48:18-46.

45. Marder E, Bucher D. Central pattern generators and the control of rhythmic movements. Curr Biol 2001;11:R986R996.

46. Musuvathy S. Coherently interacting dynamics in the neuromuscular system [doctoral dissertation]. Los Angeles: Department of Electrical Engineering, University of Southern California; 2011. Online document at: http://digitallibrary .usc.edu/cdm/compoundobject/collection/p15799coll3/id/ $195901 / \mathrm{rec} / 2$

47. Epstein D. Self-organizing systems \& pattern generation. Network Spinal Analysis: Certification Level Intensive; Hyannis, MA; October 1996.

48. Heimburg T, Jackson AD. On soliton propagation in biomembranes and nerves. Proc Natl Acad Sci U S A 2005; 102:9790-9795.

49. Andersen SS, Jackson AD, Heimburg T. Towards a thermodynamic theory of nerve pulse propagation. Progress Neurobiol 2009;88:104-113.

50. Nordenström B. Biologically closed electric circuits: clinical, experimental and theoretical evidence for an additional circulatory system. Philadelphia: Coronet Books; 1983.
51. Jonckheere E, Musuvathy S. Somatopsychic wave research demonstartion and lecture. Network Spinal Analysis Intermediate Advanced Care Intensive; Boulder, CO; January 9, 2013.

52. Epstein D. Network spinal analysis: introducing the concept of the 'spinal gateway.' Chiropractic Journal 2002;27.

53. Jones J, Bae Y. Ultrasonic visualization and stimulation of classical oriental acupuncture points. Med Acupunct 2004; $15: 24-26$.

54. Wisneski L, Anderson L. The Scientific Basis of Integrative Medicine: 2nd ed. Boca Raton, FL: CRC Press; 2009.

55. Senzon S, Epstein D, Lemberger D. The network spinal wave as a central pattern generator. In: McCoy M, ed. International Research and Philosophy Symposium; Spartanburg, SC; 2014. Ann Vertebral Subluxation Res 2015;49-98.

56. Schneider ED, Sagan D. Into the Cool: Energy Flow, Thermodynamics, and Life. Chicago: University of Chicago Press; 2005.

Address correspondence to: Simon A. Senzon, MA, DC 218 Chestnut Street Asheville, NC 28801

E-mail: simon@institutechiro.com 